\title{
A rectangular slotted waveguide array for high power microwave applications
}

\author{
Cédric MARTEL \\ ONERA/DEMR, Université de Toulouse, F-31055 Toulouse, France \\ cedric.martel@onera.fr
}

\begin{abstract}
In this paper, a rectangular slotted waveguide array is proposed for high power microwave applications. The antenna has been designed in the context of implementing a nonlethal high power transmission device onboard a drone to stop and neutralize non-cooperative vehicles. The proposed antenna comprises 16 slotted waveguides and a beamforming network, which is folded to maximize the compactness of the antenna. It has been specifically designed to send maximum EM energy in the range $20^{\circ}-30^{\circ}$ off-axis direction to avoid undesired ground reflections during operations. It has the ability to withstand a peak power of $500 \mathrm{~kW}$. A measured gain of $27.7 \mathrm{dBi}$ has been obtained at 3.15 GHz. The antenna has been successfully used to stop a car engine from a distance of approximately 9 meters.
\end{abstract}

Keywords—waveguide array; high power microwave

\section{INTRODUCTION}

For high power applications, the antenna is a key system component. It must have some dedicated characteristics such as the ability to handle the high power applied at its input and to produce a high directive beam. Additional constraints such as size and weight must also be taken into account, especially for high power systems that operate from an airborne platform like a drone.

Various antennas for high power applications have been reported these last few years. In [1], a circular shape radial line slot antenna operating at $9.42 \mathrm{GHz}$ is presented. The antenna is circularly polarized and presents an aperture efficiency of $44.8 \%$. In [2], the design of an array of one-dimensional leaky wave antennas operating at $9.6 \mathrm{GHz}$ is proposed.

In this paper, a rectangular slotted waveguide array designed for high power handling capabilities at the operating frequency of $3 \mathrm{GHz}$ is proposed. The presented antenna design represents the first step toward the implementation of an onboard drone high power microwave device.

This work has been carried out in the context of the FP 7 European research project AEROCEPTOR which aimed to develop new and innovative concepts of operation to remotely and safely control, slow, and stop non-cooperative vehicles in both land and sea scenarios, by the means of remote piloted aerial systems [3].

\section{ANTENNA REQUIREMENTS}

TABLE I. presents the requirements to be taken into account for the antenna design.
TABLE I. INITIAL REQUIREMENTS

\begin{tabular}{|c|c|}
\hline Central frequency & $3 \mathrm{GHz}$ \\
\hline Gain & $\geqslant 25 \mathrm{dBi}$ \\
\hline Pointing direction & $\sim 20^{\circ}-30^{\circ}$ off-axis \\
\hline Power handling & $500 \mathrm{~kW}$ \\
\hline Weight & As light as possible \\
\hline
\end{tabular}

III. DESCRIPTION OF THE ANTENNA

\section{A. Elementary slotted waveguide}

A rectangular slotted waveguide, acting as a leaky-wave radiating structure, defines the elementary building block of the antenna. With internal dimensions of $72.14 \mathrm{~mm} \times 34.04 \mathrm{~mm}$ (identical to the WR284 standard) and a wall thickness of $3.6 \mathrm{~mm}$, the guide comprises an input port, 20 radiating slots, and a short circuit at its end (Fig.1). To maximize the gain, the position of the radiating slots must be carefully chosen so that the power radiated by each slot is approximately the same along the waveguide length. Slots close to the waveguide input port have a larger amount of available power as compared to the slots that are further away from the input. Moreover, for a given available power in the waveguide, the EM leakage into free-space depends on the position of the radiating slots with respect to the waveguide central line. To control the power radiated by the slots, the following taper function defines the slot positions with respect to the central line:

$|y|=y_{\min }+x^{\alpha} \cdot\left\lfloor\left(y_{\max }-y_{\min }\right) /\left(x_{\max }-x_{\min }\right)^{\alpha}\right\rfloor$

where $x$ is the slot position along the waveguide, $\alpha$ the taper expansion factor and $x_{\min }, x_{\max }, y_{\min ,} y_{\max }$ initial constraints. An optimization of the taper expansion factor $\alpha$ and the slot length was carried out. Moreover, the slot periodicity along the waveguide was chosen in such a way as to obtain a backward scan angle in the range $20^{\circ}-30^{\circ}$ at $3 \mathrm{GHz}$. Simulations of the slotted waveguide were carried out with HFSS. The mutual coupling between adjacent waveguides was taken into account by considering an infinite number of slotted waveguides in the plane orthogonal to the scan direction.

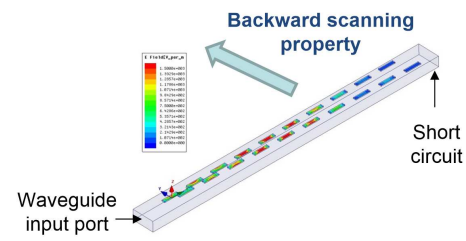

Fig. 1. HFSS model of the elementary slotted waveguide. 

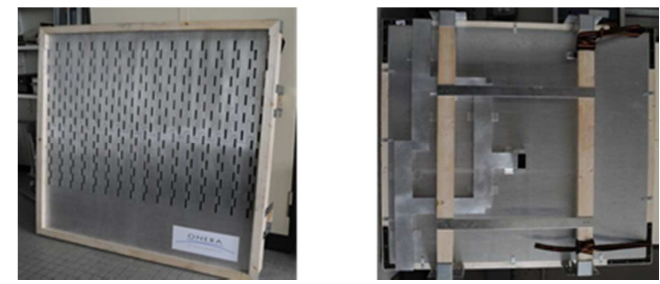

Fig. 2. Photos of the manufactured slotted waveguide array.

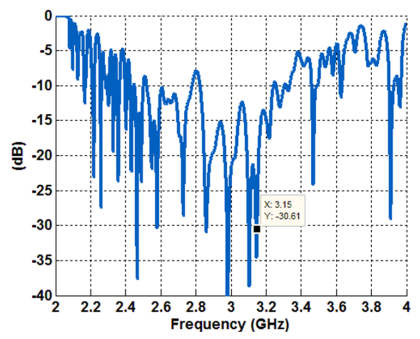

Fig. 3. Measured reflection coefficient of the array (S11 in $\mathrm{dB})$.

The optimum slots size and periodicity are $10 \mathrm{~mm} \times 49.6 \mathrm{~mm}$ and $42.6 \mathrm{~mm}$, respectively.

\section{B. Waveduide array antenna}

The antenna is comprised of 16 slotted waveguides (Fig.2), fed by a folded Beam Forming Network (BFN). The antenna has one input port characterized with a standard flange for WR284 rectangular waveguide. The BFN consists of a corporate network distributing the energy with equal amplitude and phase in the 16 waveguides via coupling slots. The folded configuration of the BFN enables the preservation of a reasonably small antenna size with respect to the operating frequency. The antenna size is $1204 \mathrm{~mm} \times 1215 \mathrm{~mm} \times 78.9$ $\mathrm{mm}$ (excluding the port).

The various antenna parts (radiating plate, waveguide walls ...) have been machined separately and fixed together with metal screws. Because of the large aperture size, the radiating plate consists of four assembled parts.

For testing purposes, a wooden frame has been built for the antenna. The antenna weights $40.5 \mathrm{~kg}$. It must be noted that the antenna weight could be dramatically reduced by reducing the waveguide wall thickness or by considering different manufacturing methods such as plastic metallization or $3 \mathrm{D}$ printing.

\section{PERFORMANCE OF THE ANTENNA}

The antenna has been tested in ONERA's anechoic chamber. The reflection coefficient of the array was measured in the band [2-4] GHz (Fig. 3). The antenna is well-matched from 2.82 to $3.24 \mathrm{GHz}$ where the reflection coefficient is below $-10 \mathrm{~dB}$. The reflection coefficient reaches $-22 \mathrm{~dB}$ at $3 \mathrm{GHz}$ and $-30.6 \mathrm{~dB}$ at $3.15 \mathrm{GHz}$. The measured gain patterns are shown in Fig. 4. The highest gain is $27.7 \mathrm{dBi}$ at $3.15 \mathrm{GHz}$, corresponding to an aperture efficiency of $43.5 \%$. Variation of the beam position can be observed in function of frequency. At $3.15 \mathrm{GHz}$, the main beam points in the $24^{\circ}$ off-axis direction.

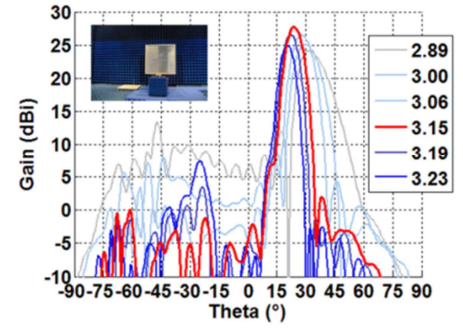

Fig. 4. Measured radiation pattern of the array.

The pattern bandwidth (frequency band where the gain is within $3 \mathrm{~dB}$ with respect to the maximum value) is $240 \mathrm{MHz}$ running from 2.99 to $3.23 \mathrm{GHz}$.

This antenna has been used successfully during an experimental campaign, which consisted of remotely stopping the engine of a non-moving car by sending a high peak power EM signal of $500 \mathrm{~kW}$ from a fix suspended platform approximately 9 meters away from the car.

\section{CONCLUSION}

A slotted waveguide array has been proposed for sending high power signals. The antenna comprises 16 slotted waveguides, which are fed by a folded beam forming network. The antenna has the capability to avoid back radiation from ground reflections by pointing its main beam at $24^{\circ}$ off-axis at $3.15 \mathrm{GHz}$. The antenna has been built and tested. A maximum antenna gain of $27.7 \mathrm{dBi}$ has been measured at $3.15 \mathrm{GHz}$, corresponding to an aperture efficiency of $43.5 \%$.

The antenna has been used successfully during an experimental campaign, which consisted of stopping the engine of a stationary car with high pulse power of $500 \mathrm{~kW}$ sent from a fixed suspended platform. Future work could investigate ways to reduce the antenna weight so that the antenna integration on the airborne platform becomes more practical. Metallized plastic or 3D printing could be interesting approaches to consider.

\section{ACKNOWLEDGMENT}

This project has received funding from the European Union's Seventh Programme for research, technological development and demonstration under grant agreement No 285144

The author would like to thank Daniel Prost and Laurent Guibert for building the antenna wooden frame; and Benjamin Gabard and Wilfrid Quenum for measuring the antenna.

\section{REFERENCES}

[1] C.W. Yuan, S.R. Peng, T. Shu, Z-Q. Li, and H. Wang, "Designs and experiments of a novel radial line slot antenna for high-power microwave application", IEEE Trans. on Antennas and Propagation, vol. 61, No. 10, Oct. 2013.

[2] L. Guo, W. Huang, C. Chang, J. Li, Y. Liu, and R. Meng, "Studies of a leaky-wave phased array antenna for high-power microwave applications", IEEE Trans. on plasma science., vol. 44, No. 10, Oct. 2016.

[3] http://www.aeroceptor.eu 